\title{
Muscle Fatigue and the Mechanisms of Task Failure
}

\author{
Sandra K. Hunter ${ }^{1,}$ Jacques Duchateau, ${ }^{2}$ and Roger M. Enoka ${ }^{3}$ \\ ${ }^{1}$ Exercise Science Program, Department of Physical Therapy, Marquette University, Milwaukee, WI; \\ ${ }^{2}$ Laboratory of Applied Biology, Université Libre de Bruxelles, Brussels, Belgium; and ${ }^{3}$ Department of \\ Integrative Physiology, University of Colorado at Boulder
}

HUNTER, S. K., J. DUCHATEAU, and R. M. ENOKA. Muscle fatigue and the mechanisms of task failure. Exerc. Sport Sci. Rev., Vol. 32, No. 2, pp. 44-49, 2004. An alternative approach in the study of muscle fatigue is to address the question, "What causes task failure during a fatiguing contraction?" This approach is described by considering how variation in the type of load supported and contraction intensity influence both the time to task failure and the centrally mediated adjustments in reflex activity and motor unit behavior. Keywords: central nervous system, electromyogram, force task, motor units, position task, reflexes

\section{INTRODUCTION: WHAT CAUSES MUSCLE FATIGUE?}

Muscle fatigue is defined as an exercise-induced reduction in the maximal force capacity of the muscle (10). The classic approach used to identify the cause of muscle fatigue has been to distinguish between neural and muscular mechanisms. This can be accomplished, for example, by applying an electrical stimulus to the peripheral nerves and either (a) comparing the decline in muscle force during a voluntary contraction with that evoked by the imposed electrical stimulation (2) or (b) quantifying the extra muscle force that electrical stimulation can elicit during a maximal voluntary effort of the muscle, which is known as the interpolated stimulus (twitch) technique (10). When the muscle force evoked by the stimulus exceeds the force that can be exerted by the voluntary contraction, the decline in voluntary force (muscle fatigue) is at least partially caused by an impairment of neural mechanisms.

Such interventions have demonstrated that both muscular and neural mechanisms are impaired during fatiguing contractions (10). However, the relative contributions of the neural mechanisms to the decline in force seem to vary with the task that is performed. To identify the adjustments that occur within the nervous system during fatiguing contrac-

Address for correspondence: Sandra K Hunter, PhD, Exercise Science Program, Department of Physical Therapy, Marquette University, P.O. Box 1881, Milwaukee, WI 53201 (E-mail: Sandra.Hunter@marquette.edu).

Accepted for publication: January 27, 2004.

0091-6331/3202/44-49

Exercise and Sport Sciences Reviews

Copyright (C) 2004 by the American College of Sports Medicine tions, studies have focused on the influence of afferent feedback, descending inputs, and spinal circuitry on the output of the motor neuron pool. For example, the metabolites that accumulate in muscle during a prolonged contraction excite afferent fibers that both enhance the central drive to maintain muscle perfusion by increasing mean arterial pressure and modulate the discharge rate of some motor neurons (10). Such observations indicate that the decline in force during a fatiguing contraction usually involves multiple neural mechanisms.

Consequently, the question "What causes muscle fatigue?" is difficult to answer. A response to the question is confounded further by the dependence of the mechanisms responsible for the decline in force on the details of the task that is performed. The task details that seem to influence the contributing mechanisms include the type and intensity of exercise, the muscle groups involved, and the physical environment in which the task is performed.

\section{A DIFFERENT QUESTION: WHAT CAUSES TASK FAILURE?}

Instead of attempting to identify a global cause of muscle fatigue, the impairment of performance resulting from an acute decline in the force capacity of muscle can be assessed by asking a more functional question, "What causes task failure?" As an example of this approach, a series of studies examined how varying the type of load supported during a fatiguing contraction with the elbow flexor muscles influences both the time to task failure and the rates of impairment of the contributing mechanisms (12-14). The task was 
to support a submaximal load (15\%-20\% of maximum) with the elbow flexor muscles for as long as possible. Subjects performed two isometric contractions in a randomized order on separate days: a force task and a position task. The force task required the subject to exert a submaximal force against a rigid restraint while the wrist was attached to a force transducer. The position task required the subject to maintain a constant elbow angle while supporting an inertial load that was equal to the force exerted during the force task. Consequently, the net muscle torque for each subject was similar for the two tasks. The time to task failure was $1402 \pm$ $728 \mathrm{~s}$ (mean \pm SD) for the force task and $702 \pm 582 \mathrm{~s}$ for the position task (14).

Despite the longer duration for the force task, the average, rectified electromyogram (EMG) of the elbow flexor muscles increased at the same rate for the two tasks, but achieved a lesser amplitude at the termination of the position task (Fig. 1A). The increase in EMG was likely dominated by the recruitment of motor units with modest changes in discharge rate $(6,11)$. The rates of increase in mean arterial pressure (Fig. 1B) and heart rate (pressor response), ratings of perceived exertion, and the fluctuations in motor output (force
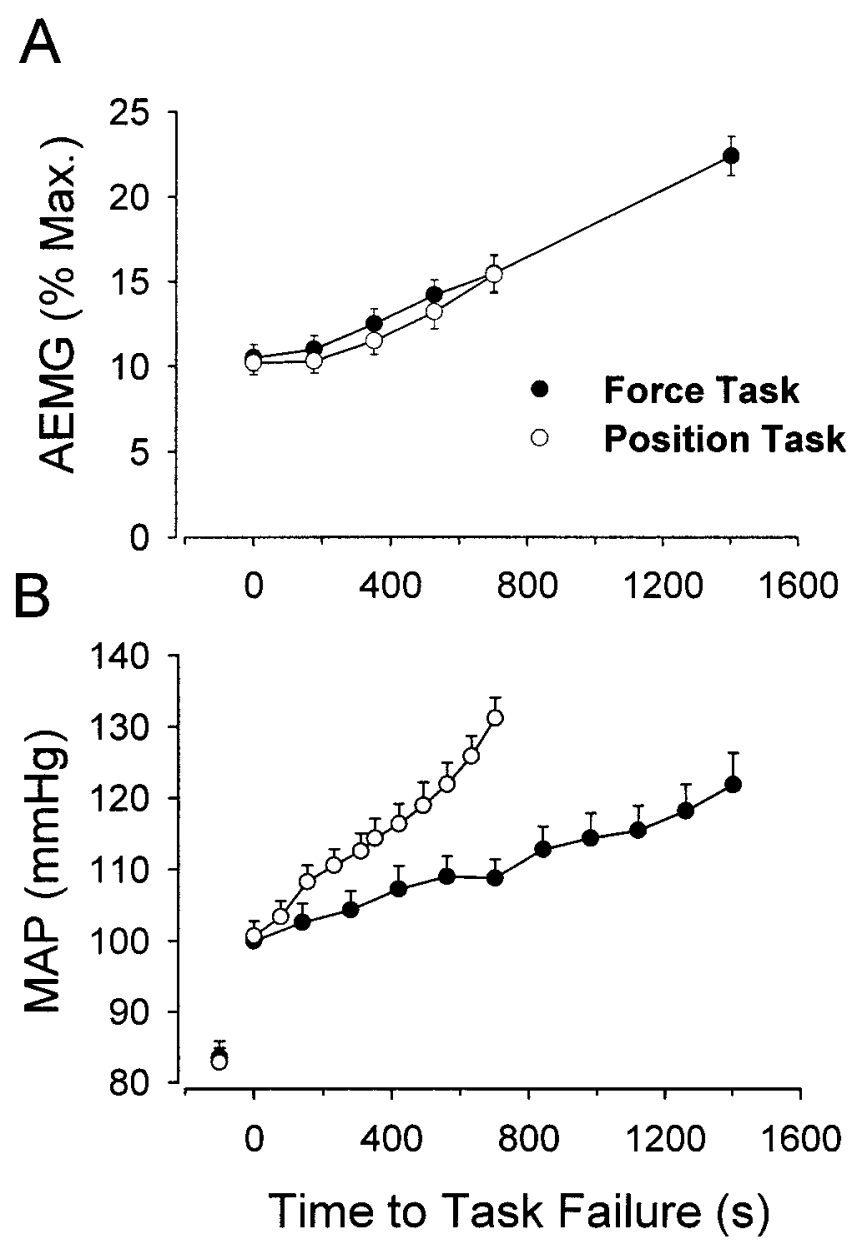

Figure 1. The averaged EMG (A) and mean arterial pressure (B) for an isometric contraction of the elbow flexor muscles sustained at $15 \%$ of maximum until task failure for a force task and position task. The rate of increase in muscle activation (EMG) was similar for the two tasks despite a greater rate of increase in perfusion pressure (MAP) during the position task. Data from (14). and acceleration) were greater for the position task, which suggested a greater rate of increase in the descending drive to the spinal motor neurons during the position task. However, the identical rates of change in EMG activity for the two tasks indicated that the net output of the motor neuron pool from the spinal cord was similar for the two tasks. Therefore, these results suggested that despite a similar net muscle torque for the two tasks, the motor neurons in the spinal cord received greater amounts of both excitatory and inhibitory input during the position task so that the output (EMG) was similar. Given the evidence of a greater rate of increase in the central neural activity during the position task, the differential inputs received by the motor neuron pool during the two tasks was likely responsible for the briefer time to task failure for the position task.

The challenge, therefore, is to define the contributions of the excitatory and inhibitory inputs to the spinal cord and their influence on task failure when the requirements of the task are varied. Although much is known about the neural input that is received by motor neurons, the functional role of the inputs during fatiguing contractions remains uncertain (10). The spinal motor neurons receive direct excitatory inputs from supraspinal sources and peripheral sensory receptors, such as the group 1a afferents of the muscle spindle. The motor neurons also can receive inhibitory input from group $1 \mathrm{~b}$ afferents (tendon organ) and excitatory input from group II (muscle spindle) afferents, but this is delivered indirectly through interneurons, and the influence of inputs from these sources are not well defined for fatiguing contractions (10). Although the input transmitted by group III-IV afferents elicits excitatory postsynaptic potentials in supraspinal centers, interposed interneurons cause the same input to evoke inhibitory postsynaptic potentials in spinal motor neurons and to mediate presynaptic inhibition of the input onto the motor neurons. Similarly, the motor neurons can receive inhibitory input through recurrent inhibition from the Renshaw cell. Furthermore, an inhibitory effect can be mediated by reducing the excitatory input, an effect known as disfacilitation; for example, presynaptic inhibition of the group 1a afferents by the group III-IV afferents as the muscle spindle afferents impinge on the motor neurons.

The progressive enlargement of the interference EMG during a sustained submaximal contraction often is considered to denote an increase in the excitatory output of the motor neurons. The increase in the interference EMG during such tasks, however, provides limited information about motor unit activity (7). It is not possible, for example, to use surface EMG to determine the relative contributions of motor unit recruitment and discharge rate to the change in performance $(6,7,11)$ or to explain why the EMG amplitude at task failure is less than that recorded during a maximal voluntary contraction (MVC) (9). Furthermore, an assessment of changes in the amplitude of the EMG signal is confounded by the presence of cancellation, which refers to the loss of signal content resulting from the overlapping positive and negative phases of the motor unit potentials (7). Cancellation cannot be accommodated completely by either the processing technique (e.g., rectification, root mean square) or normalization of the recorded signal, although the significance of this effect during different fatiguing tasks is yet 
to be identified. Consequently, the interference EMG itself provides limited information on the adjustments in motor unit activity during sustained contractions. To learn more about the changes that occur in the spinal cord during fatiguing contractions, it was necessary to characterize the modulation of reflex responses and to record the activity of individual motor units.

\section{REFLEX ACTIVITY AND TASK FAILURE}

The contribution of reductions in reflex activity to task failure has been quantified by recording the changes in the EMG evoked by a stimulus that activates the afferent fibers (stretch or electrical stimulation). Brief electrical stimulation of the muscle spindle afferents (group Ia fibers) during a low-level contraction generates a short-latency reflex, also called the Hoffmann (H)-reflex, and a long-latency reflex. The $\mathrm{H}$-reflex largely comprises monosynaptic input to the spinal motor neurons, whereas the long-latency reflex includes multiple synapses that can involve the evoked potential traveling to supraspinal centers before the output is initiated by the motor neuron. Participation of the supraspinal pathway in the long-latency reflex is more certain for upper limb muscles compared with lower limb muscles. Comparison of short- and long-latency reflexes can distinguish the involvement of spinal and supraspinal circuitry in task failure, particularly in the upper limbs (10).

A probable role for spinal mechanisms was demonstrated by a study that recorded the reflex responses elicited by quick stretches imposed before and after sustained voluntary contractions (1). There was a reduction in amplitude of the short-latency response after the sustained fatiguing contraction, with evidence to suggest that the decrease in reflex amplitude was not the result of a change in muscle spindle responsiveness, but involved an impairment of central neural mechanisms.

To assess the contributions of adjustments in the spinal and supraspinal circuitry (Fig. 2), the $\mathrm{H}$ - and long-latency reflexes were recorded before and after a sustained maximal contraction of the first dorsal interosseus muscle in the hand (5). Although the amplitude of the $\mathrm{H}$ reflex was reduced by $30 \%$ after the sustained contraction, there was no change in the amplitude of the long-latency reflex. These reductions were not attributable to an impairment of neuromuscular propagation because the reflex responses were normalized to the $\mathrm{M}$ wave (the maximal EMG response to supramaximal electrical stimulation of the peripheral nerve). In contrast, the amplitude of the long-latency reflex increased in a nonfatigued hand muscle, the abductor pollicis brevis, which suggests that there was an increase in the excitatory descending drive during the fatiguing contraction to compensate for changes in the synaptic input and the intrinsic properties of the motor neuron pool.

Further insight on the contribution of spinal circuitry to task failure was gained in a similar experiment when the $\mathrm{H}$ and long-latency reflexes were recorded during the course of isometric fatiguing contractions performed at $25 \%$ of maximum for $492 \mathrm{~s}$ and $50 \%$ of maximum for $205 \mathrm{~s}$ by the abductor pollicis brevis muscle (4). The amplitudes of both

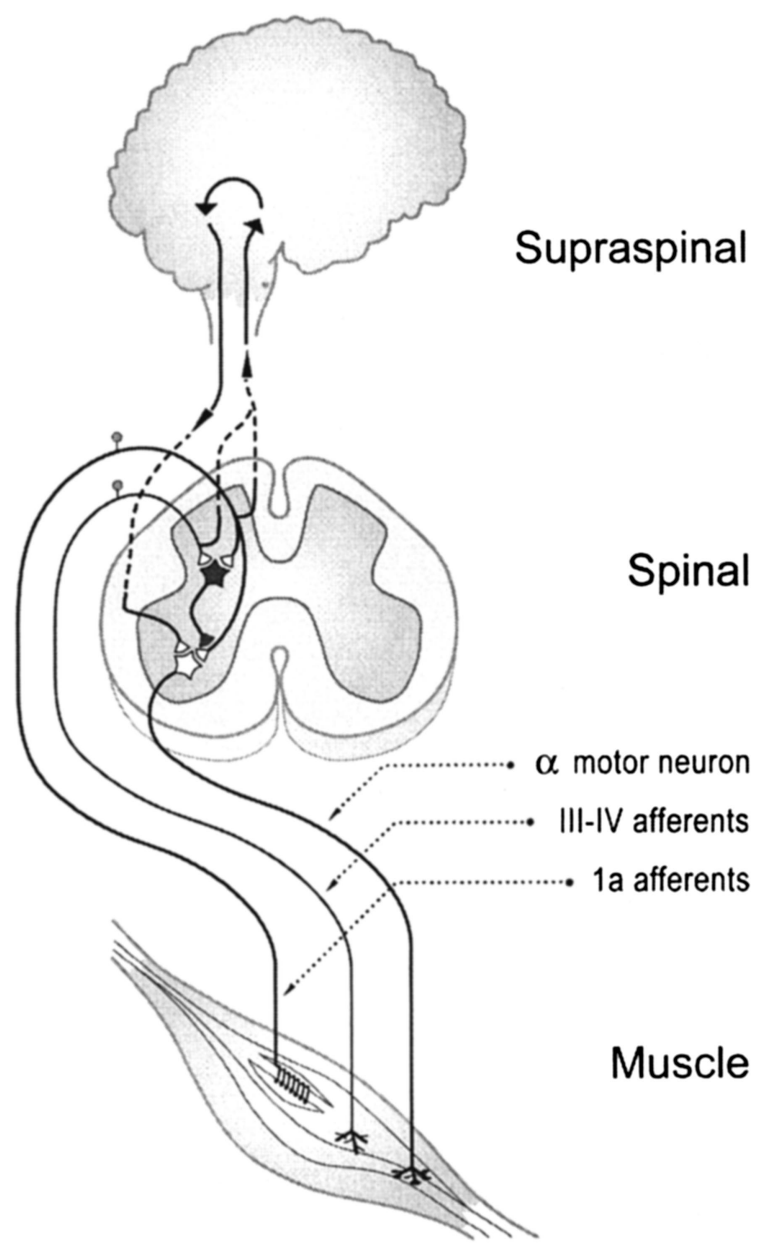

Figure 2. Schematic of the selected inputs received by motor neurons in the spinal cord. The diagram shows some of the excitatory and inhibitory pathways to the spinal cord that have been found to modulate the output of the motor neuron pool during fatiguing contractions. Although motor neurons can receive inhibitory inputs from a variety of sources that are mediated through interneurons, the diagram indicates that the dominant inhibitory effect from group III-IV afferents during fatiguing contractions involves a reduction in excitatory input by presynaptic inhibition of the group la afferents.

reflexes were reduced significantly at task failure. The decrease in $\mathrm{H}$-reflex amplitude was similar for the $25 \%$ and $50 \%$ contractions and was reduced to a comparable level observed previously for a sustained maximal contraction (5); thus, the effect was independent of contraction intensity and duration (Fig. 3). The decline in $\mathrm{H}$-reflex amplitude was caused either by enhanced inhibition of the motor neuron pool or by impaired synaptic transmission within the spinal cord. Because the decline in H-reflex amplitude developed gradually and there was no reduction in the $\mathrm{H}$-reflex amplitude for an intermittent contraction performed at $25 \%$ of maximal voluntary contraction for a similar duration, the reduced excitation observed during the sustained contraction likely originated from the group III-IV afferents. In contrast to the consistent reduction in $\mathrm{H}$-reflex amplitude at the conclusion of the sustained contractions, the amplitude of the longlatency reflex declined by $27 \%$ at the end of the contraction at $50 \%$ of maximum and decreased by $39 \%$ for the contraction at $25 \%$ of maximum. These findings suggest that the 


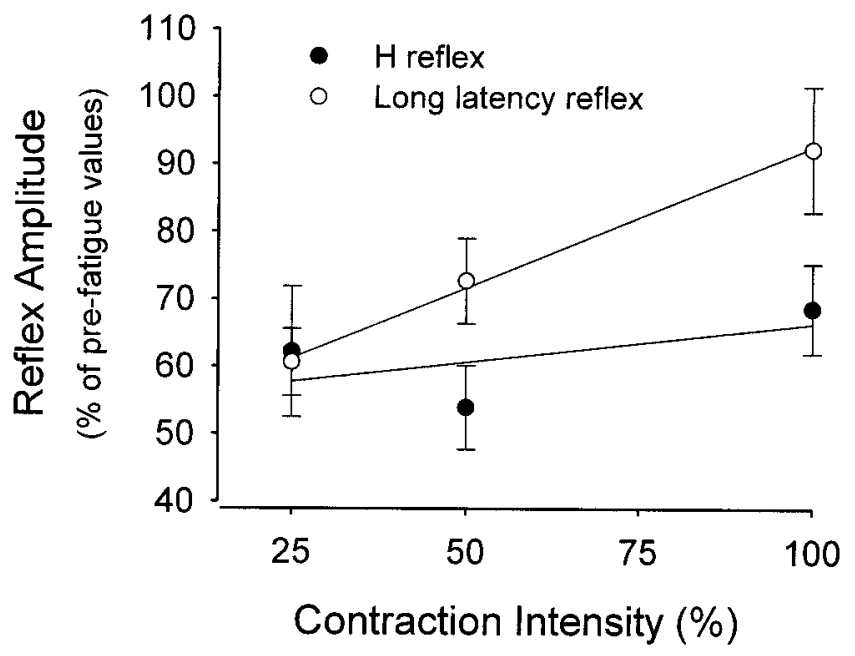

Figure 3. Changes in reflex amplitude as a function of contraction intensity during sustained fatiguing contractions with a hand muscle (abductor pollicis brevis). The mean ( \pm SEM) amplitude of the $\mathrm{H}$ reflex and the long-latency reflex at the end of contractions that were sustained at $25 \%$, $50 \%$, and $100 \%$ of the initial MVC force. The figure includes the regression lines for the $\mathrm{H}$ reflex and the long-latency reflex. There was no difference in the reduction of the H-reflex amplitude for the contractions performed at the three intensities. However, the long-latency reflex experienced a greater reduction in amplitude after the contractions at $25 \%$ and $50 \%$ compared with $100 \%$ contraction. Data from (4).

decline in excitatory input onto the motor neurons from spinal or peripheral origins was constant for sustained fatiguing contractions of varying intensities and durations, but was partially compensated by an intensity-dependent augmentation of excitatory descending drive $(4,5)$. Consequently, the modulation of reflex activity seems to indicate that changes in the balance of excitatory and inhibitory inputs onto the motor neuron pool, including the reduction in excitatory input by presynaptic inhibition, contribute to task failure.

\section{MOTOR UNIT BEHAVIOR AND TASK FAILURE}

The required muscle force during a fatiguing task is obtained by varying the number of motor units that are recruited and the rates at which the motor neurons discharge action potentials. For submaximal contractions, the interaction between the inputs to the motor neuron pool and the intrinsic properties of the motor neurons result in a progressive increase in the number of activated motor units and modest changes in discharge rate. For example, the discharge rates of the motor units that were recruited at the beginning of a fatiguing contraction with the first dorsal interosseous muscle decreased continually over the course of a series of intermittent submaximal contractions, whereas those motor units recruited later during the task exhibited a nonmonotonic increase and then decrease in discharge rate (3). The decrease in discharge rate was most prominent in the firstrecruited motor units (recruitment threshold $<25 \% \mathrm{MVC}$ ). To sustain the target force, therefore, additional motor units were recruited to compensate for the loss of force resulting from the leftward shift of the operating point on the forcefrequency relation by the motor units that were already active
(8). In addition, muscle force was influenced by the initial increase and subsequent decrease in the discharge rates of later-recruited motor units. Such divergent behavior of the low- and high-threshold motor units probably is not a consequence of the descending drive to the motor neuron pool, but likely represents a time-dependent property of the motor neurons.

The activity of single motor units also changes when the task is varied. This was evident in a study that recorded the discharge of the same motor units in biceps brachii muscle during the performance of the force and position tasks (15). Motor units with low ( $n=20$; range, $3 \%-21 \%$ MVC) and moderate $(n=12$; range, $26 \%-44 \%$ MVC) recruitment thresholds were examined when the force was sustained at $3.5 \pm 2.1 \%$ of the maximal voluntary force above the recruitment threshold of the isolated motor unit. The initial discharge rate of the motor units (low- and moderate-threshold combined) was approximately $14 \mathrm{~Hz}$ in both tasks and declined to $12.5 \pm 2.7 \mathrm{~Hz}$ for the force task and to $10.9 \pm 2.6$ $\mathrm{Hz}$ for the position task. Furthermore, discharge rate variability (coefficient of variation) began at approximately 19\% for both tasks and increased to $21 \%$ at the end of the force task and to $25 \%$ at the end of the position task (Fig. 4). Differences in the discharge rate of the same motor units in the two tasks were accompanied by a pronounced recruitment of additional motor units during the position task compared with the force task. Furthermore, the enhanced motor unit activity during the position task was associated with greater increases in the fluctuations in motor output, mean arterial pressure, heart rate, and ratings of perceived

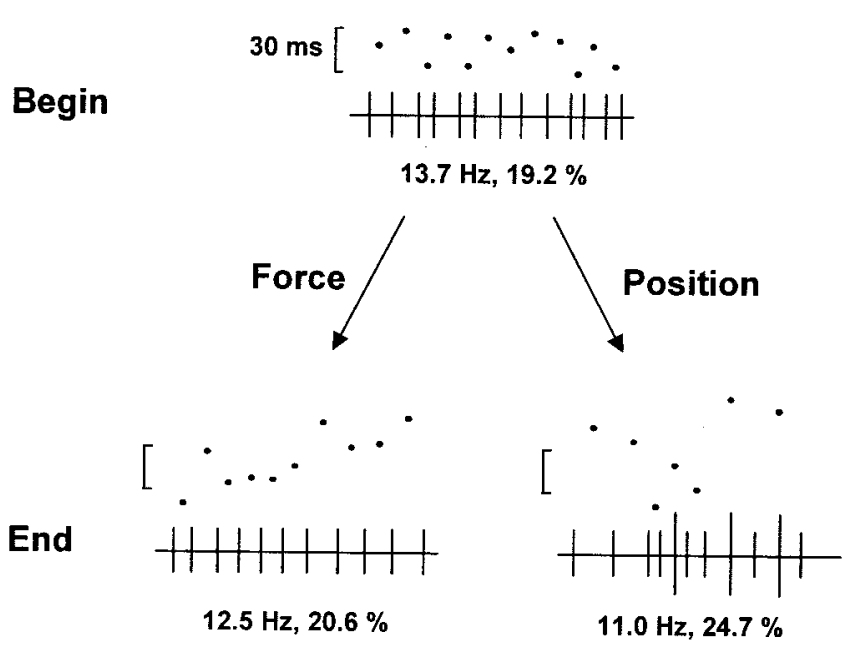

Figure 4. Schematic changes in the mean discharge rate of the same motor unit in the biceps brachii muscle for the force and position tasks. The discharge of the action potentials is shown at the beginning (top panel) and the end (bottom panels) of each task performed for the same duration $(161 \pm 96 \mathrm{~s})$. The time between successive action potentials, which corresponds to the instantaneous discharge rate, is indicated as dots above the trains of action potentials in each panel. The initial discharge rate $(13.7 \mathrm{~Hz})$ and the coefficient of variation for discharge rate $(19.2 \%)$ were similar for the two tasks, which underscores the similarity in the mechanical output for the two tasks. At the end of the position task, however, the average discharge rate was lower and the coefficient of variation for discharge rate was greater for the position task compared with the force task. Note the recruitment of an additional motor unit during the position task. Data from (15). 


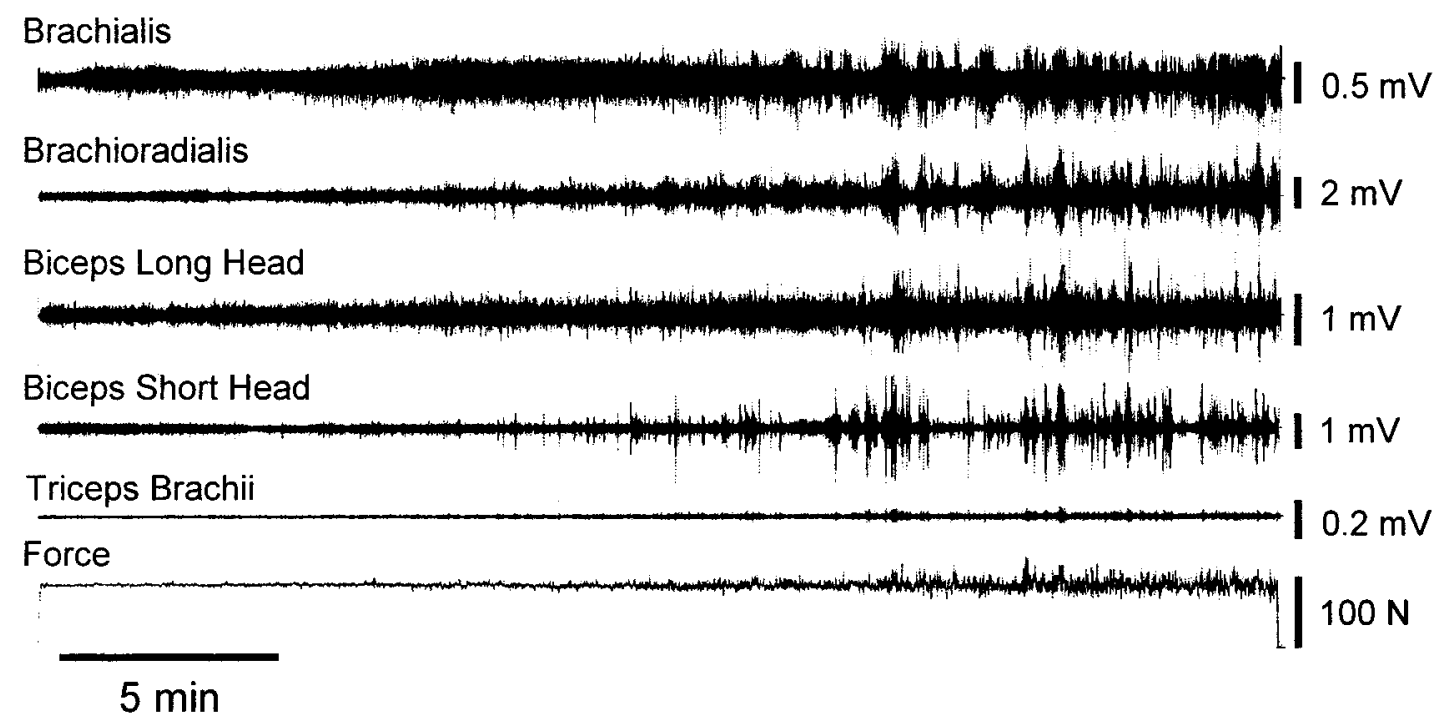

Figure 5. Representative data for the force task sustained to failure. The target force was $20 \%$ of the maximal voluntary contraction force. The interference EMG of the elbow flexors (top four traces) increased progressively throughout the contraction with significant bursting in the middle and latter stages of the contraction. There was minimal EMG activity in the antagonist muscle, triceps brachii. The average force (bottom trace) remained constant, but the fluctuations increased in amplitude toward the end of each contraction. (Reprinted from Hunter, S.K., and R.M. Enoka. Changes in muscle activation can prolong the endurance time of a submaximal isometric contraction in humans. J. Appl. Physiol. 94:108-118, 2003. Copyright @ 2003 The American Physiological Society. Used with permission.)

exertion. These findings are consistent with the interpretation that the position task is associated with greater excitation of the motor neuron pool, which likely contributed to the briefer time to task failure observed in the previous studies (12-14).

In addition to the progressive modulation of motor unit recruitment and discharge rate, sustained submaximal contractions also can involve the transient recruitment of motor units. Such behavior was evident in the recordings of single motor unit activity during a low-force fatiguing contraction ( $10 \%$ of maximum), but not when the target force was $40 \%$ of maximum (6). The presence of bursts of EMG activity in the interference signal (Fig. 5) also can be used as an index of the transient activation of motor units (12-14). Observations indicate that the EMG bursts are not present at the onset of the contraction, but begin to appear as the contraction proceeds toward task failure. As the burst rate increases, the force recording becomes more tremulous (bottom trace in Fig. 5). Furthermore, increases in the time to task failure, such as can occur with practice of the force task, were associated with a prolongation of the time to the first appearance of the EMG bursts (12). Similarly, a briefer duration for a fatiguing contraction, such as the time to task failure for the position task compared with the force task (Fig. 1 ), was associated with an earlier onset of EMG bursts (14). Presumably, the EMG bursts indicate the transient recruitment of motor units to compensate for the decline in force capacity of the continuously active motor units.

The influence of changes in motor unit activity on the time to task failure can be estimated by examining the effects of various interventions, such as practice and limb immobilization. With practice of the force task, some subjects increased the time to task failure (responders), whereas others did not (nonresponders) (12). The prolongation of the time to task failure for the responders was accompanied by a decrease in the rate of rise in the average EMG, a delay in the transient recruitment of the motor units (EMG bursts), and a reduced rate of increase in the force fluctuations. In contrast, practice did not alter the time to task failure for the position task (13). The two studies indicate, therefore, that increases in the duration of a task are accompanied by corresponding reductions in the rate of increase in motor unit activity. Furthermore, the capacity for an adaptation in motor unit activity with practice differed for the two types of loads, which underscores the initial observation that the excitatory and inhibitory input received by the motor neuron pool differs for these two tasks despite a similar mechanical output.

\section{SUMMARY AND FUTURE DIRECTIONS}

The mechanisms that contribute to muscle fatigue depend on the task that is performed, which has been known for several decades. To emphasize the functional role of these adjustments, however, an alternative strategy is to focus on the mechanisms that are responsible for task failure. Even relatively subtle variations in task can be associated with marked differences in the time to task failure. For example, variation in the intensity and type of load supported during a contraction seems to involve differences in the balance of excitatory and inhibitory spinal inputs to the motor neuron pool and corresponding changes in the time to task failure. Future studies should examine the specific mechanisms responsible for the differences in the force and position tasks and should expand the performance domain to include such task variables as the muscle group performing the task, the duty cycle of the contraction, and contraction type. 


\section{Acknowledgments}

This work supported in part by National Institute of Neurological Disorders and Stroke (grant no. R01 NS43275 [RME]) and by Fonds National de la Recherche Scientifique of Belgium (JD). The authors thank Jeffrey Rainwater (Fig. 2) and Carol Mottram (Fig. 4) for their contributions to the review.

\section{References}

1. Balestra, C., J. Duchateau, and K. Hainaut. Effects of fatigue on the stretch reflex in a human muscle. Electroencephalogr. Clin. Neurophysiol. 85:46-52, 1992.

2. Bigland-Ritchie, B., F. Furbush, and J.J. Woods. Fatigue of intermittent submaximal voluntary contractions: central and peripheral factors. J. Appl. Physiol. 61:421-429, 1986.

3. Carpentier, A., J. Duchateau, and K. Hainaut. Motor unit behaviour and contractile changes during fatigue in the human first dorsal interosseus. J. Physiol. 534:903-912, 2001.

4. Duchateau, J., C. Balestra, A. Carpentier, and K. Hainaut. Reflex regulation during sustained and intermittent submaximal contractions in humans. J. Physiol. 541:959-967, 2002.

5. Duchateau, J., and K. Hainaut. Behaviour of short and long latency reflexes in fatigued human muscles. J. Physiol. 471:787-799, 1993.

6. Fallentin, N., K. Jørgensen, and E.B. Simonsen. Motor unit recruitment during prolonged isometric contractions. Eur. J. Appl. Physiol. 67:335341, 1993.
7. Farina, D., R. Merletti, and R.M. Enoka. The extraction of neural strategies from the surface EMG. J. Appl. Physiol. 96:1486-1495, 2004.

8. Fuglevand, A.J., and D.A. Keen. Re-evaluation of muscle wisdom in the human adductor pollicis using physiological rates of stimulation. J. Physiol. 549:865-875, 2003.

9. Fuglevand, A.J., K.M. Zackowski, K.A. Huey, and R.M. Enoka. Impairment of neuromuscular propagation during human fatiguing contractions at submaximal forces. J. Physiol. 460:549-572, 1993.

10. Gandevia, S.C. Spinal and supraspinal factors in human muscle fatigue. Physiol. Rev. 81:1725-1789, 2001.

11. Garland, S.J., R.M. Enoka, L.P. Serrano, and G.A. Robinson. Behavior of motor units in human biceps brachii during a submaximal fatiguing contraction. J. Appl. Physiol. 76:2411-2419, 1994.

12. Hunter, S.K., and R.M. Enoka. Changes in muscle activation can prolong the endurance time of a submaximal isometric contraction in humans. J. Appl. Physiol. 94:108-118, 2003.

13. Hunter, S.K., R. Lepers, C.J. MacGillis, and R.M. Enoka. Activation among the elbow flexor muscles differs when maintaining arm position during a fatiguing contraction. J. Appl. Physiol. 94:2439-2447, 2003.

14. Hunter, S.K., D.L. Ryan, J.D. Ortega, and R.M. Enoka. Task differences with the same load torque alter the endurance time of submaximal fatiguing contractions in humans. J. Neurophysiol. 88:30873096, 2002.

15. MacGillis, C.J., J.G. Semmler, J.M. Jakobi, and R.M. Enoka. Motor unit discharge differs with intensity and type of isometric contraction performed with the elbow flexor muscles. Med. Sci. Sport Exer. 35:S280, 2003. 